\title{
Déterminer les indicateurs de qualité médicaux avec des données de facturation
}

\author{
FMH/santésuisse: \\ groupe de travail «Qualité»* \\ * Membres \\ (ordre alphabétique): \\ Yvonne Andrista ${ }^{\mathrm{a}}$, \\ Christoph Bosshard ${ }^{\mathrm{b}}$, \\ Karl Hampl ${ }^{\text {, }}$ \\ René Kühne ${ }^{a}$, \\ Varja Meyer ${ }^{\mathrm{b}}$, \\ Felix Roth ${ }^{\mathrm{a}}$, \\ Stefan Teskea, \\ Jürg von Below ${ }^{\mathrm{b}}$ \\ a santésuisse \\ b FMH
}

\section{Contexte}

En vertu des art. 58 et 59 LAMal et de l'art. 77 OAMal, une convention existe entre la FMH et santésuisse depuis octobre 2009 dans le but d'élaborer conjointement des concepts concernant l'assurance et le contrôle de la qualité des prestations médicales. Egalement sur une base contractuelle, les deux partenaires ont ensuite mis en place un groupe de travail conjoint dédié à la qualité (GT FMH/santésuisse). Celui-ci a décidé d'axer son premier projet commun sur le respect de l'art. 22a let. f LAMal, qui prévoit que les fournisseurs de prestations mettent les données relatives à la qualité de leurs prestations médicales à la disposition des autorités fédérales au moyen d'indicateurs de qualité médicaux.

\section{Interactions médicamenteuses}

Tout d'abord, le groupe de travail a procédé à l'évaluation de travaux internationaux existants sur les indicateurs de qualité dans le domaine ambulatoire en se référant à un catalogue de critères compilé par ses soins, et en se concentrant principalement sur les indicateurs qualité élaborés en Allemagne**. Suite à ces travaux de base, le groupe de travail a obtenu une liste d'indicateurs considérés comme pertinents par la FMH et santésuisse pour l'évaluation de la qualité en médecine. Dans une étape suivante, le groupe de travail FMH/santésuisse a estimé les différents indicateurs selon leur pertinence, leur applicabilité et leur investissement (en termes de coûts, de temps, etc.). Après avoir pesé les principaux avantages et inconvénients, le groupe de travail a décidé d'approfondir ses recherches sur les indicateurs utilisés dans le domaine des interactions médicamenteuses, notamment en raison de l'accès des assureurs-maladie à des données sur les médicaments pratiquement exhaustives, très bien détaillées, structurées et disponibles sous forme électronique. L'inconvénient, comme dans beaucoup d'autres domaines, réside dans l'absence de données cliniques structurées (archivage électronique) issues de la pratique ambulatoire.

Alors que les données du domaine choisi offrent une bonne disponibilité, celles relevées présentent un grand intérêt car leur analyse bénéficie aux patients, aux médecins et aux répondants des coûts. Par ailleurs, l'analyse de telles données répond à la proposition de concrétisation de la stratégie Qualité de la Confédération qui estime que 30 à $50 \%$ de toutes les erreurs de traitement sont liées à la médi- cation. Il existe ainsi un besoin urgent d'agir rapidement en matière de sécurité et de médication [1].

\section{Données de routine}

A l'heure actuelle, dans le secteur ambulatoire, la collecte de données cliniques représente un investissement que les cabinets médicaux ne sont pas en mesure d'assumer avec leurs ressources faute d'un manque d'utilisation de dossiers électroniques de patients. Dans la droite lignée du rapport de concrétisation relatif à la stratégie de la Confédération en matière de qualité, le groupe de travail FMH/santésuisse a décidé de procéder à une étude de faisabilité et d'examiner si les indicateurs de qualité dans le domaine des interactions médicamenteuses pouvaient être déterminés sur la base de données de routine. Selon les assureurs-maladie, cette procédure pourrait fonctionner dans le domaine choisi car plusieurs d'entre eux répertorient quasiment tous les médicaments prescrits par les médecins en ambulatoire, et remboursés dans le cadre de l'assurance obligatoire des soins, en utilisant un pharmacode. Cela permet de retrouver systématiquement la substance active, la quantité et la forme galénique des médicaments facturés, ainsi que la fréquence à laquelle ils sont prescrits ou remis.

Après avoir mis le projet au concours, le groupe de travail $\mathrm{FMH} /$ santésuisse a finalement confié la réalisation de l'étude de faisabilité au Basel Institute for Clinical Epidemiology and Biostatistics (CEB). Nous vous résumons ci-après les données du problème, la méthode employée ainsi que les résultats de l'analyse. Ceux qui souhaitent approfondir la question peuvent commander l'étude par courriel à asqm[at] fmh.ch. Le CEB rédigera également un travail scientifique dans lequel il publiera les résultats épidémiologiques.

\section{Données du problème}

L'objectif de l'étude est d'analyser s'il est possible de définir des indicateurs de qualité grâce aux données des assureurs-maladie concernant la facturation des médicaments dans le secteur des soins médicaux ambulatoires. Ces indicateurs devraient être définis de telle sorte qu'ils permettent de quantifier de manière fiable et valide la sécurité en matière de médication (concrètement, de savoir si les médicaments ont été prescrits correctement) au niveau des différents fournisseurs de prestations - c'est en effet le degré de précision exigé par l'art. 22a let. f LAMal. 


\section{Méthode}

L'étude ne se base pas sur des événements cliniques occasionnés par des interactions médicamenteuses par manque de données cliniques - mais sur les interactions potentielles de deux médicaments prescrits en même temps et dont l'interaction présente des effets indésirables cliniquement significatifs. En d'autres termes, les indicateurs qu'il s'agit de développer sont censés évaluer le processus qui englobe la prescription des médicaments et favorise l'apparition d'une interaction.

\section{Conditions-cadres}

Ces indicateurs doivent refléter le mieux possible la qualité réelle de la prescription médicamenteuse. C'est pourquoi il a fallu que le développement de ces indicateurs prenne en compte le fait que, dans des cas isolés, les patients peuvent tolérer sans problème une interaction médicamenteuse et qu'il est tout à fait possible d'avoir des interactions médicamenteuses sous contrôle grâce notamment à une surveillance adaptée et à un éventuel changement de dosage. En outre, il n'est pas toujours possible d'éviter les interactions. En l'occurrence, un médecin peut sciemment courir le risque d'une interaction potentielle car il estime que la prescription de deux ou plusieurs médicaments susceptibles d'interagir peut être plus bénéfique au patient qu'une éventuelle interaction. Il a également fallu tenir compte du fait que plusieurs médecins peuvent prescrire des médicaments à un seul et même patient et que les médecins traitent des collectifs de patients différents du point de vue du nombre, de l'âge et de la morbidité des patients. En outre, l'absence de données cliniques ne permet pas de prendre en compte la question de savoir si les médicaments prescrits sont pris confor-

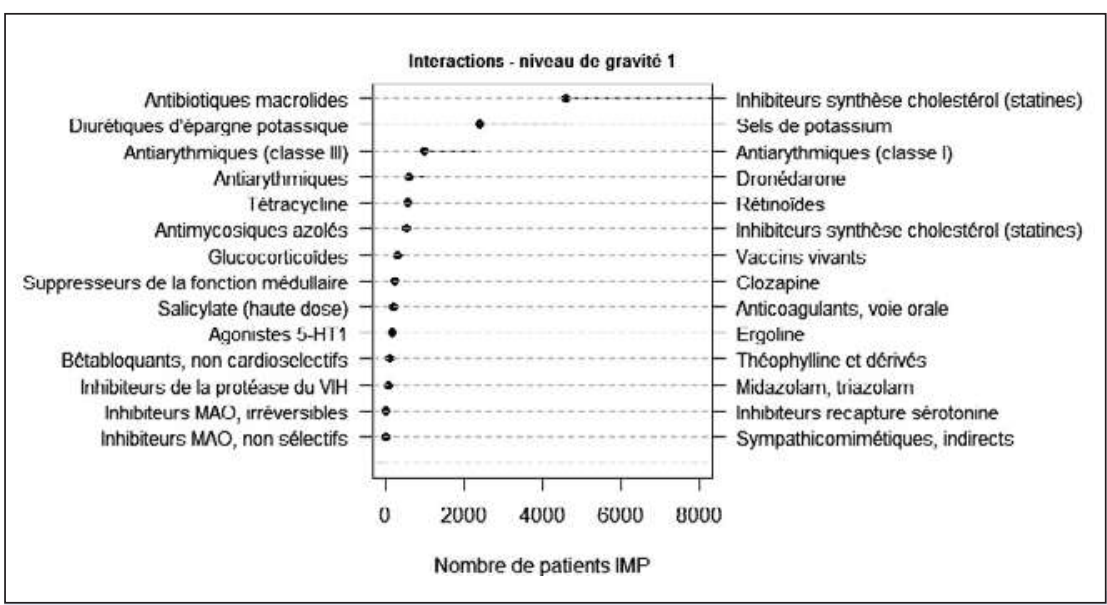

Figure 1

Nombre de personnes avec au moins une interaction médicamenteuse potentielle, degré de gravité 1 (contre-indiqué). Echantillon: 3,13 millions de personnes, période d'observation: 2010. Source: Basel Institute for Clinical Epidemiology and Biostatistics (2012): Etude de faisabilité concernant un indicateur des interactions médicamenteuses dans le secteur ambulatoire. Version brève. mément à l'ordonnance (dosage et heures) ou si la médication a été adaptée (nouvelle ordonnance).

\section{Données disponibles et indicateurs de qualité}

Dans le respect de la protection des données, l'étude a analysé sous forme anonyme les prestations médicales, les médicaments et les examens de laboratoire pris en charge par trois groupes d'assurance (CSS, Helsana et Sanitas) pour l'année 2010, soit un total de 3,13 millions d'assurés à l'assurance obligatoire, ce qui représente $40 \%$ de la population. Le CEB a créé un indicateur basé sur le nombre d'interactions médicamenteuses potentielles (IMP) pour 41 classes d'interaction qualifiées de «contre-indiquées» ou «contre-indiquées par précaution». Cet indicateur considère aussi bien le nombre de patients avec au moins une IMP que le nombre de classes d'interaction dans lesquelles un médecin présente un résultat statistique significatif. Pour faire face aux différences entre les collectifs de patients traités par les médecins (nombre de patients, leur âge et leur morbidité), on a créé une valeur de référence équivalant à un sous-collectif de patients. Ce dernier représente les patients qui s'exposent véritablement à une IMP. A titre d'exemple, dans la classe d'interaction «anticoagulants et analgésiques», le sous-collectif regroupe tous les patients qui ont eu recours au moins une fois à un coagulant pendant la période d'observation.

\section{Résultats épidémiologiques}

Parmi les 3,13 millions d'assurés associés à l'étude, $1,34 \%$ d'entre eux ont eu recours au moins une fois en 2010 à une association de médicaments qualifiée de «contre-indiquée» (degré de gravité 1) ou de «contre-indiquée par précaution» (degré de gravité 2 ). En ne considérant que les personnes de 70 ans et plus, cette proportion est nettement plus élevée et atteint respectivement $3,78 \%$ et $4,4 \%$ pour les femmes et les hommes. Les figures 1 et 2 donnent une représentation graphique des personnes présentant au moins une IMP par classe d'interaction. Parmi 20720 médecins, les indicateurs relevés ont permis de recenser 457 praticiens avec un comportement statistiquement significatif en matière de prescription de médicaments. Cela correspond à $2,2 \%$. Il est cependant difficile de comparer ces données avec les résultats d'autres pays dans la mesure où les études similaires se basent sur des définitions totalement différentes de l'interaction médicamenteuse et pondèrent différemment le degré de gravité.

\section{Evaluation de la faisabilité}

Les indicateurs de qualité ainsi développés permettent d'identifier les médecins qui, d'un point de vue statistique, prescrivent fréquemment des associations de médicaments présentant un risque potentiel d'interaction. Mais cela ne permet pas de donner un avis sur la qualité de la prescription des médicaments. En effet, d'un point de vue médical, 


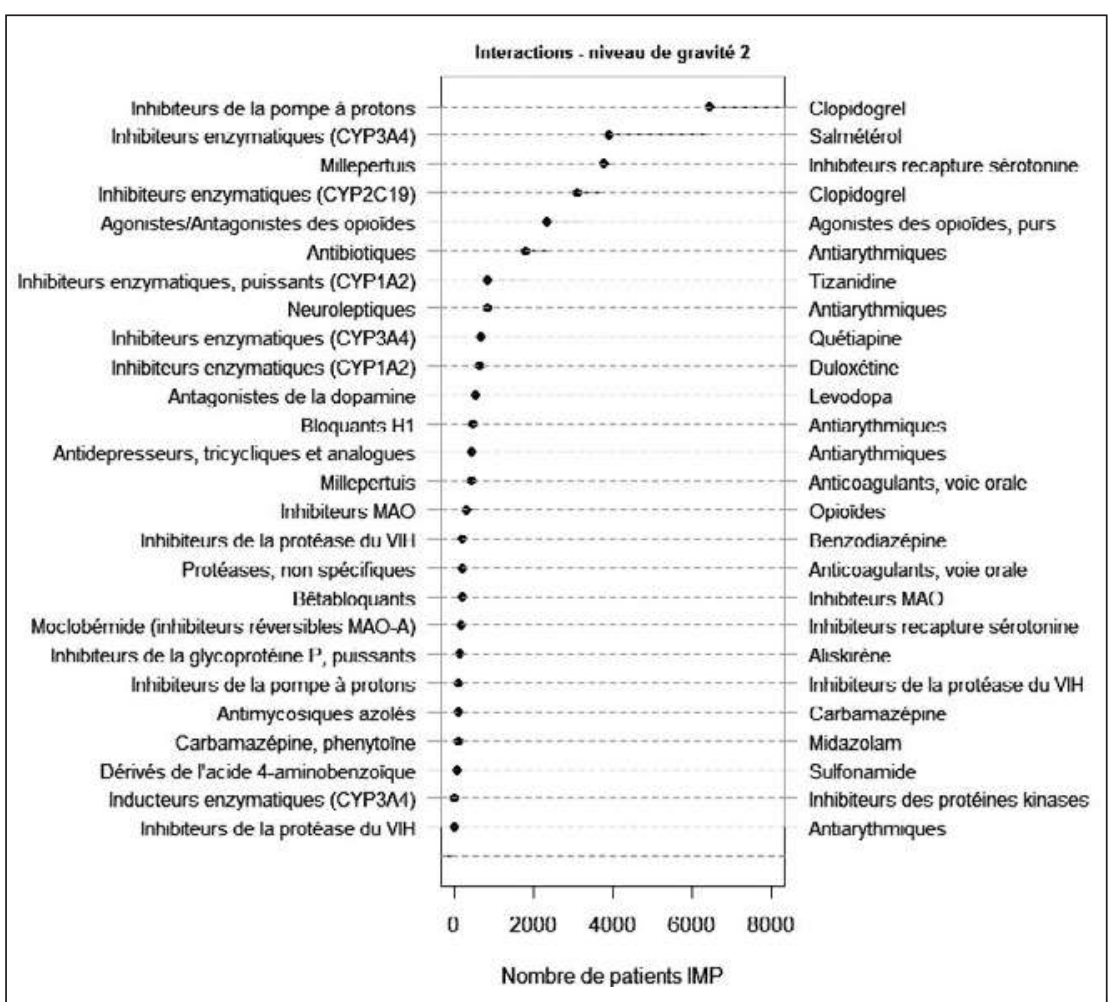

Figure 2

Nombre de personnes avec au moins une interaction médicamenteuse potentielle, degré de gravité 2 (contre-indiqué par précaution). Echantillon: 3,13 millions de personnes, période d'observation: 2010. Source: Basel Institute for Clinical Epidemiology and Biostatistics (2012): Etude de faisabilité concernant un indicateur des interactions médicamenteuses dans le secteur ambulatoire. Version brève. pects supplémentaires de la sécurité en matière de médication, comme notamment le contrôle des ordonnances de médicaments qui ne devraient pas être prescrits aux personnes âgées (critères de Beers, liste de priscus), l'emploi réduit d'antibiotiques conformément aux guidelines et les erreurs de dosage.

Enfin, les indicateurs qualité développés dans le cadre de cette étude ne s'appliquent qu'à une partie des médecins, alors que l'indicateur devrait englober tous les médecins pour répondre à l'exigence de l'art. 22 a let. f LAMal.

\section{Prochaines étapes}

L'étude de faisabilité montre qu'il est impossible de définir des indicateurs de qualité valides uniquement sur la base des données des assureurs-maladie pour ce qui est des interactions médicamenteuses. La tentative de valider les indicateurs développés en incluant des données supplémentaires dans le monitorage et la surveillance (par ex. données des examens médicaux ou des consultations de contrôle) ne s'est pas soldée par un succès. Une telle validation doit impérativement prendre en compte les données cliniques concernant les interactions médicamenteuses survenues réellement. Suite à des explorations approfondies de la faisabilité, le CEB estime qu'une validation clinique ne peut pas être réalisée dans les circonstances rencontrées actuellement dans la pratique quotidienne.

Aujourd'hui, le groupe de travail FMH/santésuisse se livre à une réflexion de fond pour savoir quelle direction donner à ses travaux à venir. Une option consisterait à travailler sur un concept en vue de créer les conditions-cadres nécessaires à la mise à disposition des données cliniques pour formuler des indicateurs de qualité dans le secteur ambulatoire.

\section{Références}

1 Office fédéral de la santé publique: Rapport au Conseil fédéral sur la concrétisation de la stratégie fédérale en matière de qualité dans le système de santé. 2011. www.bag.admin.ch/themen/krankenversicherung/00300/00304/ (Etat 20.3.2013).

2 E-mediat AG Schönbühl. Pharmavista - information for healthcare professionals; 2011. Online: www. pharmavista.ch/content/default.aspx

Vous avez la possibilité de commander le rapport final «Etude de faisabilité concernant un indicateur des interactions médicamenteuses dans le secteur ambulatoire» auprès de l'Académie suisse pour la qualité en médecine (ASQM) soit par voie électronique (asqm[at]fmh.ch) soit par téléphone au 03135911 11. Le rapport est uniquement disponible en allemand. 